\title{
A Theorem Concerning the Positive Metric
}

\author{
By \\ DEREK W. RoBinson \\ Max-Planck-Institut für Physik und Astrophysik, München
}

\begin{abstract}
It is proved that if the $n$-point correlation functions of a system vanish for all $n>N$ then they vanish for all $n>2$. The theorem is valid for a wide variety of formalisms and an explicit proof is given for a Bose system with the canonical commutation relations; a proof is sketched out for a relativistic field theory of the Wightman type. The essential property used in the proof is the positive definite metric.
\end{abstract}

\section{Introduction}

During the last few years a great deal of effort has been applied to the analysis of the mathematical structure of relativistic field theory (see for instance [1], [2]) and a number of general results have been obtained, some of which are valid for other physical theories. One property which is assumed in many theories, but which has been scarcely analysed, is the property of the positive definiteness of the metric in Hilbert space. It is the purpose of the present note to prove one simple theorem which is essentially a direct consequence of this property alone. This theorem is concerned with the hierarchy of functions describing the correlations between various points or events of the system, the so-called truncated functions or correlation functions. Physically one would expect that the correlation between $n$ events would be small for $n$ large and consequently that it might be reasonable to construct models, described by a finite number of functions, by setting all the truncated functions for $n>N$ equal to zero, where $N$ is arbitrarily large but finite. The present theorem proves that such models are inconsistent with the positive metric unless the remaining truncated functions $n>2$ also vanish identically. Thus the class of models obtained by this physical Ansatz contains only the well known "generalized free field", which has of course been extensively applied to the many body problem and widely analysed in relativistic field theory.

In Sec. 2 of this paper we introduce several general definitions and results and show that for a system with no internal structure the above mentioned theorem is a direct consequence of a result due to MARCINKIEWICZ. In Sec. 3 this result is extended to the case of a Bose system 
with the canonical commutation relations and a proof for a relativistic field theory of the Wightman type is indicated. Sec. 4 comprises a short summary and discussion.

\section{General formulation}

A large variety of physical theories may be mathematically summarized as consisting of an algebra $\mathfrak{A}$ generated by certain elements $Q_{i}$ (fields, observables, etc.). Equations of motion, invariance properties and other physical conditions define the internal structure of the algebra, which for our present purposes is irrelevant. Ultimately one is interested in a cyclic representation of the algebra in a Hilbert space and it is well known (see for instance [3]) that corresponding to every positive linear functional $W(Q)$ over $\mathfrak{A}$ there exists such a representation. Thus to examine the cyclic representations of $\mathfrak{A}$ we need only to examine the functionals $W(Q)$ defined for all $Q \in \mathfrak{A}$ and having the two properties

$$
W\left(\lambda_{1} Q_{1}+\lambda_{2} Q_{2}\right)=\lambda_{1} W\left(Q_{1}\right)+\lambda_{2} W\left(Q_{2}\right)
$$

where $\lambda_{1}, \lambda_{2}$ are constants and $Q_{1}, Q_{2} \in \mathfrak{A}$ and

$$
W\left(Q^{+} Q\right) \geqq 0 .
$$

This result can be easily understood if we use the physical notation introduced by the definition

$$
W(Q)=\langle 0|Q| 0\rangle
$$

where $|0\rangle$ is understood to be the cyclic state in Hilbert space. (In most applications $|0\rangle$ is the ground state or the vacuum.) It is perhaps important to realize that (1) and (2) are the only properties which $W(Q)$ must have in order to be interpretable as the expectation value of $Q$ in some state.

The truncated functions

$$
W^{T}(Q)=\langle 0|Q| 0\rangle_{T}
$$

are defined from the ground state expectation values of products of the $Q_{i}$ recursively through the formula

$$
\left\langle 0\left|Q_{i_{1}} Q_{i_{2}} \ldots Q_{i_{n}}\right| 0\right\rangle=\Sigma\left\langle 0\left|Q_{i_{i}} \ldots\right| 0\right\rangle_{T} \ldots\left\langle 0\left|\ldots Q_{i_{l}}\right| 0\right\rangle_{T}
$$

where the sum is over all possible clustering of the $Q_{i_{k}}$ and the order within each cluster is carried over from the left hand side.

We now consider a system with no internal structure described by an Abelian algebra generated by a single hermitian element $Q$. In this case a generating function exists for the ground state expectation values,

$$
E(x)=\left\langle 0\left|e^{i x Q}\right| 0\right\rangle=\sum_{n=0}^{\infty} \frac{i^{n} x^{n}}{n !} W_{n}
$$


and (3) simplifies to the identification

$$
E(x)=\exp \left\{\sum_{n=1}^{\infty} \frac{i^{n} x^{n}}{n !} W_{n}^{T}\right\} .
$$

The function $E(x)$ has three relevant properties; it is bounded,

and

$$
E^{*}(x)=E(-x)
$$

$$
\int d x d y f^{*}(x) E(x-y) f(y) \geqq 0,
$$

for all $f(x)$ from some suitable class. These properties are a consequence of (2) and the hermiticity of $Q$ and they serve to define, in the terminology of probability theory, a characteristic function. We now use a theorem* due to MarcinkIEwiCz [4].

Theorem 1. If $\exp \{P(x)\}$, where $P(x)$ is a polynomial, is a characteristic function then it follows that $P(x)=i a x-b x^{2}$ where $a$ and $b$ are real and $b>0$.

A proof of this theorem is reproduced by Richten [5]. Thus, from (4), we see that if $W_{n}^{T}=0$ for all $n>N$ then $E(x)$ is a characteristic function of the type considered by the theorem and we must then conclude that $W_{n}^{T}=0$ for all $n>2$. In the next section we extend this result to more complicated systems.

\section{Applications \\ a) Bose system}

Consider a Bose system defined in terms of a field $\varphi(x)$ and its Hermitian conjugate $\varphi^{+}(x)$ which satisfy the canonical commutation relations, i.e.

$$
\left[\varphi(x), \varphi^{+}(y)\right]=\delta(x-y) \quad \text { etc. }
$$

and from which we may construct the well defined operator $\varphi(f)$ given by

$$
\varphi(f)=\int d^{3} x \varphi(x) f(x) \text { where } f(x) \in \mathfrak{S} .
$$

Let us now define

and

$$
Q_{i}=\varphi\left(f_{i}\right) \text { for } i=1,2, \ldots, n
$$

$$
Q_{i}=\varphi^{+}\left(f_{i}\right) \text { for } i=n+1, \ldots, n+m .
$$

From (3) and (6) we define the truncated functions

$$
W_{n m}^{T}\left(P_{i} ; f_{i}\right)=\left\langle 0\left|Q_{i}, \ldots, Q_{i n+m}\right| 0\right\rangle_{T}
$$

$\star$ In the course of the present work this theorem was conjectured and proved by the present author. However, before publication, it was pointed out by Dr. SCHLIEDER that the theorem had been already given by MARCINKIEWICZ [4]. The author is indebted to Dr. SCHLIEDER for this information. 
where $P_{i}$ denotes the permutation $\left\{i_{1}, \ldots, i_{n+m}\right\}$ of the set of indices $\{1, \ldots, n+m\}$. We need next

Lemma. The truncated functions $W_{n m}\left(P_{i} ; f_{i}\right)$ are independent of the permutation $P_{i}$ except for the case $n=m=1$ where

$$
\left\langle 0\left|\varphi\left(f_{1}\right) \varphi^{+}\left(f_{2}\right)\right| 0\right\rangle_{T}=\left\langle 0\left|\varphi^{+}\left(f_{2}\right) \varphi\left(f_{1}\right)\right| 0\right\rangle_{T}+\left(f_{1}, f_{2}\right) .
$$

This Lemma is a direct consequence of (5) and may be easily proved by induction, using (3). As a result of the Lemma we introduce the simplified notation

$$
W_{n m}^{T}\left(P_{i} ; f_{i}\right)=W_{n m}^{T}\left(f_{i}\right) .
$$

We are now in a position to prove

Theorem 1a. If the truncated functions $W_{n m}^{T}\left(f_{i}\right)$ have the property that they vanish identically for all $n, m$ such that $n+m>N>2$ and every choice of the test functions $f_{i}(x)$ then it follows from positive definiteness and the commutation relations that they have this property for all $n, m$ such that $n+m>2$.

Proof. Define the operator $Q$ by

$$
Q=\sum_{r=1}^{n+m}\left(e^{-i \alpha_{r}} Q_{r}+e^{i \alpha_{r}} Q_{r}^{+}\right)
$$

where the $\alpha_{r}$ are real numbers. By assumption

$$
W_{M}^{T}=\left\langle 0\left|Q^{M}\right| 0\right\rangle_{T}=0 \text { for all } M>N
$$

and as a result of theorem 1 positive definiteness implies that (8) must also be valid for $M>2$ and all $\alpha_{r}$. Therefore

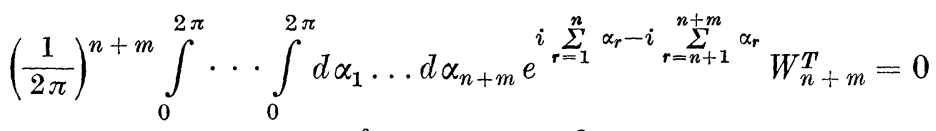

$$
\begin{aligned}
& \text { for } n+m>2 \text {. }
\end{aligned}
$$

Using (7) we calculate explicitly from (9) that

$$
\sum_{P_{i}} W_{n m}^{T}\left(P_{i} ; f_{i}\right)=0 \text { for } n+m>2 .
$$

The Lemma then allows us to conclude that

$$
W_{n m}^{T}\left(f_{i}\right)=0 \text { for } n+m>2 .
$$

As the choice of test functions and the value of $n$ in (6) are arbitrary the proof of the theorem is complete.

\section{b) Relativistic field theory}

Consider a relativistic field theory of the Wightman type defined in terms of a field $A(x)$ or $A(f)$ where

$$
A(f)=\int d^{4} x A(x) f(x) \quad \text { and } \quad f(x) \in \mathfrak{S} .
$$


The properties which are required for $A(x)$ are

1. Locality; $[A(x), A(y)]=0$, for $(x-y)^{2}<0$,

2. Lorentz invariance,

3. Positive energy

from which properties we may prove ${ }^{\star}$

Theorem $1 \mathrm{~b}$. If the truncated functions

$$
W_{n}^{T}(A)=\left\langle 0\left|A\left(f_{1}\right) \ldots A\left(f_{n}\right)\right| 0\right\rangle_{T}
$$

vanish for all $n>N>2$ then they vanish for all $n>2$.

Proof. We give an indication of the proof which starts from consideration of the operator

$$
Q=\sum_{r=1}^{N} \alpha_{r} A\left(f_{r}\right)
$$

where $\alpha_{r}$ are real parameters and the test functions $f_{i}(x)$ are chosen so that the support of each function is space-like to the support of all the remaining test functions. Now by an argument similar to that used in the proof of theorem $1 \mathrm{a}$, and using the property of locality we find immediately that

$$
\left\langle 0\left|A\left(f_{1}\right), \ldots, A\left(f_{n}\right)\right| 0\right\rangle_{T}=0 \text { for all } n>2
$$

and with the above mentioned restrictions upon the supports of the test functions. In order to remove these restrictions we need the well known analyticity properties of the Wightman functions which follow from the properties of Lorentz invariance and positive energy [1], [2]. We do not elaborate further upon this latter stage of the proof.

\section{Summary and conclusions}

The result of the present work is of a rather negative nature. It does demonstrate however the type of difficulties that might be encountered in attempting to find approximate solutions of physical problems. If one approaches the problem from a Green's function point of view it might be tempting to make approximations reducing the infinite set of coupled differential equations to a finite set but our result shows that great care must be taken, in such an approach, to avoid violating positive definiteness. The solution of an "interacting" problem is infinitely more complicated than the solution provided by a free field or "generalized" free field. In the former an infinite number of functions are involved and although one would suspect that the majority of these are small one may not ignore them completely.

Although the present work does not apply directly to a Fermi system, due to the complications introduced by anti-commutation, it

$\star$ This theorem is probably well known to most workers in the field but the only published result [6] uses much stronger restrictions upon $A(x)$. 
should be possible to construct a proof of a similar result for such a system.

The author would like to thank Prof. HAAG, Dr. Schlieder and Messrs. RINke and Roos for helpful discussions and the Aspen Institute for Humanistic Studies for their hospitality during summer 1964.

\section{References}

[1] Wightman, A., and R. F. Streater: P. C. T. Spin and Statistics and All That. New York: Benjamin 1964.

[2] Jost, R.: General Theory of Quantized Fields.Am. Math. Soc. Publications (1965).

[3] Neumark, M. A.: Normierte Algebren. Berlin: VEB Deutscher Verlag der Wissenschaften 1959.

[4] Marcinkiewicz, J.: Math. Z. 44, 612-618 (1939).

[5] RichteR, H.: Wahrscheinlichkeitstheorie. p. 300. Berlin-Göttingen-Heidelberg: Springer-Verlag 1956.

[6] Greenberq, O. W., and A. L. Licht: J. Math. Phys. 4, 613 (1963).

(Received January 1, 1965) 